\title{
Revisión sistemática sobre habilidades de pensamiento en el aula $(2000-2013)^{*}$
}

\author{
Systematic review of thinking skills in the classroom (2000-2013) \\ Revisão sistemática de habilidades de pensamento em sala de aula (2000-2013)
}

Johny Villada Zapata, ${ }^{a}$ Liliana Chaves Castaño, ${ }^{b}$ Claudia Jaramillo Ospina ${ }^{c}$

${ }^{a}$ Universidad de Antioquia

Telf.: 2198762. Correo electrónico: johnyvillada@gmail.com

bUniversidad de Antioquia

Telf.: 2198762. Correo electrónico: clasesliliana@gmail.com

${ }^{\mathrm{c}}$ Fundación Cinde

Telf.: 4448424. Correo electrónico: milenajarospina@gmail.com

\begin{abstract}
RESUMEN
Desde la segunda mitad del siglo XX, hay un interés creciente por el uso de metodologías de aprendizaje que evalúen y estimulen el desarrollo de habilidades de pensamiento en el aula, en vez de la transmisión vertical de contenidos específicos. El presente estudio retoma 60 artículos académicos publicados entre 2000 y 2013 que tenían como tema central las habilidades de pensamiento, analizando su nivel de evidencia y grado de recomendación según los lineamientos del Scottish Intercollegiate Guidelines Network (SIGN). Como hallazgos principales se encontró que la mayoría de estudios siguen enfoques empírico-analíticos y diseños cuasi experimentales. Hay una gran variedad de programas de estimulación y de instrumentos de recolección de información que dificultan un análisis sistemático de los programas. Son muy diversas las muestras utilizadas y la población intervenida y, finalmente, la mayoría de estudios se ubican en niveles moderados de evidencia y grados de recomendación.
\end{abstract}

Palabras clave: habilidades, pensamiento, aula.

\section{ABSTRACT}

Since the second half of the twentieth century, there has been a growing interest in the use of learning methods to assess and encourage the development of thinking skills in the classroom instead of the vertical transmission of a specific content. This study takes 60 academic articles published between 2000 and 2013 which had thinking skills as the central topic and analyzes their level of evidence and grade of recommendation following the Scottish Intercollegiate Guidelines Network (SIGN). The main findings were: most studies are developed with empirical analytical approaches and quasi-experimental designs; there are a variety of stimulation programs and data collection instruments that hinder a systematic analysis of programs; there are very different samples and intervened population used; and, finally, most studies are located at average levels of evidence and grades of recommendation.

Key words: skills, thinking, classrooms.

El presente artículo es producto del proyecto de investigación Aprende a Aprender: Pensamiento en movimiento. Número de acta 2012-54 del 27 de noviembre de 2012 del Centro de Investigación en Ciencias Sociales y Humanas CISH. Universidad de Antioquia. Financiado por Genesis Fundation y la Fundación Cinde Colombia. 


\section{INTRODUCCIÓN}

Optimizar el proceso de aprendizaje es quizá el desafío más importante que enfrenta la educación formal. Facilitar que los estudiantes en distintos momentos evolutivos puedan adquirir, utilizar y transformar el conocimiento acumulado de la humanidad, es la tarea más importante a la que se enfrenta un maestro, una institución educativa, un sistema de formación y una nación interesada en la educación de los individuos.

Son muchos los modelos educativos que han surgido para dar respuesta a la necesidad antes planteada. No son pocas las polémicas en torno a cómo educar y mejorar el proceso de enseñanza-aprendizaje. Dentro de esos modelos educativos, diversos autores han planteado que la clave del proceso es promover en los estudiantes habilidades de pensamiento que les permitan razonar sobre situaciones complejas, tomar decisiones utilizando distintas fuentes de información y solucionar problemas de forma lógica aprovechando el conocimiento y los contenidos a los que son expuestos en el aula.

Los modelos educativos centrados en las habilidades de pensamiento, han tenido alta difusión y son abundantes los reportes de investigación y reflexión en el tema. Sin embargo, una alta producción académica no es necesariamente equivalente a calidad y rigurosidad en los enunciados teóricos y propuestas aplicadas. Es por ello que la presente investigación rastreó los artículos académicos producidos entre el 2000 y 2013 que tenían como tema central las habilidades de pensamiento en el contexto de un aula de clase, para analizar su nivel de evidencia siguiendo los lineamientos de una revisión sistemática.

La revisión sistemática es una metodología de investigación que surge precisamente para hacer un análisis crítico de la sobreproducción académica en un tema particular. Dentro de las áreas que comprenden dicha revisión crítica, se encuentra la clasificación de los artículos según su nivel de evidencia, es decir, según la rigurosidad de los diseños de investigación y los controles aplicados al proceso, de modo que es posible establecer unos grados de recomendación que indican la calidad del texto. Así, la presente investigación busca aportar a la discusión sobre el énfasis que se ha puesto en el uso y promoción de las habilidades de pensamiento en el aula, teniendo como variable central de análisis, las metodologías usadas para abordar el tema.

\section{MÉTODO}

\subsection{TIPO DE ESTUDIO}

En una categorización general, los estudios documentales se clasifican en tres tipos: investigación documental de revisión, revisión sistemática y meta análisis. El presente artículo corresponde un estudio de revisión sistemática, es decir, un trabajo de síntesis que reporta, entre otras cosas, el nivel de evidencia que tienen las investigaciones sobre un tema particular en un periodo de tiempo limitado, permitiendo hallar tendencias investigativas.

\subsection{FUENTES DOCUMENTALES}

Las bases de datos de las cuales fueron extraídos los estudios de la presente investigación fueron: Ebsco - Psychology \& Behavioral Sciences Collection, Red de Revistas Científicas 
de América Latina y el Caribe, España y Portugal Redalyc, Dialnet y Science Direct. Para la búsqueda los criterios de inclusión fueron: publicaciones en revistas indexadas entre 2000 y 2013, artículos provenientes de la psicología, la pedagogía y sus intersecciones, habilidades de pensamiento como tema central de los estudios. Los criterios de exclusión fueron: publicaciones en revistas no indexadas con fechas anteriores a 2000, boletines, reseñas de libros, comentarios o perspectivas que no se deriven de investigaciones.

\subsection{INSTRUMENTOS}

Los artículos científicos fueron analizados siguiendo las directrices de la Scottish Intercollegiate Guidelines Network (SIGN). Esta clasificación busca analizar críticamente la gran cantidad de publicaciones científicas con base a la rigurosidad de sus diseños, haciendo una clasificación que jerarquiza los estudios revisados en cualquier tema/ problema. (Ver Tabla 1).

Tabla 1. Clasificación de las recomendaciones en función del nivel de evidencia.

Scottish Intercollegiate Guidelines Network

\section{NIVELES DE EVIDENCIA}

I.a La evidencia científica procede de meta-análisis de estudios controlados y aleatorios o revisiones sistemáticas.

I.b La evidencia científica procede de, al menos, un estudio controlado y aleatorio.

II.a La evidencia científica procede de, al menos, un estudio prospectivo controlado, bien diseñado y sin aleatorizar.

II.b La evidencia científica procede de, al menos, un estudio cuasi experimental, bien diseñado.

III La evidencia científica procede de estudios descriptivos no experimentales, bien diseñados, como estudios comparativos, de correlación o de casos y controles

IV La evidencia científica procede de documentos u opiniones de expertos y/o experiencias clínicas de autoridades de prestigio

\section{GRADOS DE RECOMENDACIÓN}

A Corresponde a los niveles de evidencia científica I.a y I.b. Existe una evidencia 'buena' con base en la investigación para apoyar la recomendación.

B Corresponde a los niveles de evidencia científica II.a, II.b y III. Existe una evidencia 'moderada' con base en la investigación para apoyar la recomendación.

C. Corresponde al nivel de evidencia IV. La recomendación se basa en la opinión de expertos o en un panel de consenso. 


\subsection{PROCEDIMIENTO}

Se hicieron búsquedas hasta el 30 de octubre de 2013 para seleccionar los artículos y hacer un fichaje inicial. Para la búsqueda se usaron las siguientes palabras clave Skills, Thinking, Classroom y sus equivalentes en español. Una vez que se registraron los artículos en una ficha de ingreso de información, se procedió a la clasificación de su nivel de evidencia y los grados de recomendación para, posteriormente, crear una base de datos en SPSS 21 que permitió analizar la frecuencia de las categorías de construidas.

\section{RESULTADOS}

Se revisó un total de 60 artículos de los cuales la mayoría fueron publicados en idioma inglés. Hubo una proporción similar de textos encontrados en las bases de datos siendo Ebscohost y Science Direct las que mayor cantidad aportaron. Además, el periodo de tiempo en el que se agrupan la mayoría de ellos es entre 2005 y 2009 (Ver Tabla 2).

Tabla 2. Idioma de las publicaciones, bases de datos donde se encontraron los textos y año de publicación

\begin{tabular}{lccc}
\hline Variable de Análisis & Descripción de la variable & Frecuencia & Porcentaje \\
\hline \multirow{3}{*}{ Idioma de la publicación } & Español & 13 & $21,7 \%$ \\
& Inglés & 47 & $78,3 \%$ \\
& Total & 60 & $100,0 \%$ \\
\hline \multirow{3}{*}{ Base de datos donde } & Dialnet & 6 & $10,0 \%$ \\
se encontró el artículo & Redalyc & 6 & $10,0 \%$ \\
& Ebscohost & 24 & $40,0 \%$ \\
& Science Direct & 24 & $40,0 \%$ \\
& Total & 60 & $100,0 \%$ \\
Año de la publicación & Entre 2000 y 2004 & 12 & $20,0 \%$ \\
& Entre 2005 y 2009 & 26 & $43,3 \%$ \\
& Entre 2010 y 2013 & 22 & $36,7 \%$ \\
& Total & 60 & $100,0 \%$ \\
\hline
\end{tabular}

La mayoría de trabajos encontrados fueron de tipo empírico analítico, seguido por estudios documentales y una menor proporción de estudios mixtos, cualitativos y de metaanálisis. En cuanto a los diseños de investigación, se encontró con mayor frecuencia estudios cuasi experimentales y de revisión documental. También hubo estudios transversales, longitudinales, hermenéuticos, meta-análisis y de investigación acción participación, pero en menor proporción (Ver Tabla 3). 
Tabla 3. Tipo de estudio y diseño de investigación

\begin{tabular}{|c|c|c|c|}
\hline Variable de Análisis & Descripción de la variable & Frecuencia & Porcentaje \\
\hline \multirow{6}{*}{ Tipo de estudio } & Empírico Analítico & 30 & $50,0 \%$ \\
\hline & Documental & 21 & $35,0 \%$ \\
\hline & Mixto & 5 & $8,3 \%$ \\
\hline & Cualitativo & 2 & $3,3 \%$ \\
\hline & Meta-análisis & 2 & $3,3 \%$ \\
\hline & Total & 60 & $100,0 \%$ \\
\hline \multirow{9}{*}{$\begin{array}{l}\text { Diseño de investigación } \\
\text { del estudio }\end{array}$} & Cuasi experimental & 23 & $38,3 \%$ \\
\hline & Revisión documental & 21 & $35,0 \%$ \\
\hline & Medición Transversal & 7 & $11,7 \%$ \\
\hline & $\begin{array}{l}\text { Cuasi experimental- } \\
\text { longitudinal }\end{array}$ & 4 & $6,7 \%$ \\
\hline & Meta-análisis & 2 & $3,3 \%$ \\
\hline & Hermenéutico & 1 & $1,7 \%$ \\
\hline & $\begin{array}{l}\text { Investigación Acción } \\
\text { Participación }\end{array}$ & 1 & $1,7 \%$ \\
\hline & Longitudinal & 1 & $1,7 \%$ \\
\hline & Total & 60 & $100,0 \%$ \\
\hline
\end{tabular}

De las investigaciones que no eran documentales, la mayoría de ellas presentaban algún programa de estimulación de las habilidades de pensamiento y una menor proporción hacían un trabajo descriptivo sobre ellas. Dentro de las primeras, los autores presentan en su mayoría programas creados por ellos mismos y son menos los trabajos sobre programas ya establecidos y validados anteriormente (como por ejemplo el de Enriquecimiento Instrumental o el de Filosofía para Niños de Lipman). El diseño específico de investigación más usado en las publicaciones que tenían algún programa de estimulación fue ABA con grupo control, además de un tipo de exposición por fuera del currículo (Ver Tabla 4). 
Tabla 4. Abordaje de las habilidades de pensamiento, programa de intervención aplicado, diseño de investigación específico y tipo de exposición

\begin{tabular}{|c|c|c|c|}
\hline Variable de Análisis & Descripción de la variable & Frecuencia & Porcentaje \\
\hline \multirow{3}{*}{$\begin{array}{l}\text { Abordaje de habilidades } \\
\text { de pensamiento }\end{array}$} & $\begin{array}{l}\text { Descripción de las } \\
\text { habilidades }\end{array}$ & 11 & $28,2 \%$ \\
\hline & Programas de estimulación & 28 & $71,8 \%$ \\
\hline & Total & 39 & $100,0 \%$ \\
\hline \multirow{7}{*}{$\begin{array}{l}\text { Programa de intervención } \\
\text { aplicado }\end{array}$} & $\begin{array}{c}\text { Programa creado por los } \\
\text { autores }\end{array}$ & 20 & $71,4 \%$ \\
\hline & Enriquecimiento Instrumental & 3 & $10,7 \%$ \\
\hline & $\begin{array}{l}\text { Cognitive Acceleration } \\
\text { through Science Education' } \\
\text { (CASE) }\end{array}$ & 2 & $7,1 \%$ \\
\hline & Filosofia Para niños Lipman & 1 & $3,6 \%$ \\
\hline & Programa Coral & 1 & $3,6 \%$ \\
\hline & $\begin{array}{l}\text { Activating children's thinking } \\
\text { skills (ACTS) }\end{array}$ & 1 & $3,6 \%$ \\
\hline & Total & 28 & $100,0 \%$ \\
\hline \multirow{4}{*}{$\begin{array}{l}\text { Diseño de investigación } \\
\text { especifico de aplicación } \\
\text { del programa }\end{array}$} & ABA con grupo control & 18 & $64,3 \%$ \\
\hline & ABA sin grupo control & 9 & $32,1 \%$ \\
\hline & $\begin{array}{l}\text { Investigación Acción } \\
\text { Participación }\end{array}$ & 1 & $3,6 \%$ \\
\hline & Total & 28 & $100,0 \%$ \\
\hline \multirow{4}{*}{ Tipo de exposición } & Fuera del currículo & 13 & $46,4 \%$ \\
\hline & $\begin{array}{l}\text { Completamente en el } \\
\text { currículo }\end{array}$ & 8 & $28,6 \%$ \\
\hline & Medianamente en el currículo & 7 & $25,0 \%$ \\
\hline & Total & 28 & $100,0 \%$ \\
\hline
\end{tabular}

Continuando con los artículos en que los autores presentan algún programa de intervención, se observa lo siguiente: la mayor parte de ellos usaron un tiempo de exposición inferior a 6 meses, son las pruebas de pensamiento, psicométricas y de inteligencia los instrumentos más usados para evaluar los resultados, la población en la que más se intervino fue estudiantes de secundaria, y el número de personas que participaron en los programas osciló, en su mayoría, entre 1 y 100 personas, seguido por entre 100 y 500 (Ver Tabla 5). 
Tabla 5. Tiempo de exposición, instrumentos de recolección de información, población y número de personas intervenidas

\begin{tabular}{|c|c|c|c|}
\hline Variable de Análisis & Descripción de la variable & Frecuencia & Porcentaje \\
\hline \multirow{6}{*}{ Tiempo de exposición } & Menos de 6 meses & 15 & $53,6 \%$ \\
\hline & Entre 6 meses y un año & 2 & $7,1 \%$ \\
\hline & Entre 1 y 2 años & 3 & $10,7 \%$ \\
\hline & Más de dos años & 3 & $10,7 \%$ \\
\hline & No reporta & 5 & $17,9 \%$ \\
\hline & Total & 28 & $100,0 \%$ \\
\hline \multirow{9}{*}{ Instrumentos de evaluación } & Análisis de tarea & 4 & $14,3 \%$ \\
\hline & Encuesta & 2 & $7,1 \%$ \\
\hline & observación y entrevistas & 1 & $3,6 \%$ \\
\hline & Pruebas de conocimientos & 1 & $3,6 \%$ \\
\hline & Pruebas de inteligencia & 6 & $21,4 \%$ \\
\hline & Pruebas de pensamiento & 7 & $25,0 \%$ \\
\hline & Pruebas psicométricas & 6 & $21,4 \%$ \\
\hline & $\begin{array}{c}\text { Pruebas psicométricas y } \\
\text { entrevistas }\end{array}$ & 1 & $3,6 \%$ \\
\hline & Total & 28 & $100,0 \%$ \\
\hline \multirow{7}{*}{ Población intervenida } & Preescolar & 1 & $3,6 \%$ \\
\hline & Primaria & 6 & $21,4 \%$ \\
\hline & Secundaria & 17 & $60,7 \%$ \\
\hline & Universitarios & 2 & $7,1 \%$ \\
\hline & Docentes & 1 & $3,6 \%$ \\
\hline & No reporta & 1 & $3,6 \%$ \\
\hline & Total & 28 & $100,0 \%$ \\
\hline \multirow{6}{*}{$\begin{array}{l}\text { Número de personas } \\
\text { intervenidas }\end{array}$} & Entre 1 y 100 & 12 & $42,9 \%$ \\
\hline & Entre 100 y 500 & 11 & $39,3 \%$ \\
\hline & Entre 500 y 1000 & 1 & $3,6 \%$ \\
\hline & Más de 1000 & 3 & $10,7 \%$ \\
\hline & No informa & 1 & $3,6 \%$ \\
\hline & Total & 28 & 100,0 \\
\hline
\end{tabular}


Finalmente, siguiendo los lineamientos de la Scottish Intercollegiate Guidelines Network, la mayoría de artículos se encuentran en gran B de recomendación, distribuidos de la siguiente manera. En nivel de evidencia II.a se encuentran los trabajos de: Barak, BenChaim y Zoller (2007); Demir (2008); McLellan (2006); y el de Sanz de Acedo Lizarraga et al., (2009). En nivel de evidencia II.b se encuentran los trabajos de: Adey, Robertson y Venville (2002); Aizikovitsh y Amita (2010); Altintas y Sukru Ozdemir (2012); Athman Ernst y Monroe (2004); Burke y Williams (2008), Burke y Williams (2012a); Cerillo Martin (2002); Cuicas et al. (2007); Dewey y Bento (2009); Garcia-Moriyon et al. (2000); Kaufman y Burden (2004); Kozulin et al., (2010); Larraz y Allueva (2012); Mbano (2003); Olaoye (2012); Raiziene y Grigaite (2005); Rendón Uribe (2011); Rezaei et al. (2013a); Rezaei et al. (2013b); Saleh (2009); Sokol et al. (2008); West (2003); y el de Ya-Ting, YaChin Chuang y Shin-Shang (2012). En nivel de evidencia III los artículos de: Abdullaha y Osmanb (2010); Beltran y Torres (2009); Blattner y Frazier (2002); Borjas y De la Peña Leyva (2009); Bronwen y Howe (2003); Delicea et al. (2009); Deniz y Kinkal (2009); Sarigoz (2012); Tuncer y Ozeren (2012); y el de Ulas, Epcacan y Kocak (2012).

Después de los artículos ubicados en el grado de recomendación $\mathrm{B}$, se encuentran los que están en grado de recomendación C (nivel de evidencia IV). Esos textos son los siguientes: Amestoy de Sanchez (2002); Bayona Vargas (2003); Beyer (2008); Black (2012); Burke y Williams (2012b); Cherian, Rao y Srivastava (2013); Colcotta, Russell y Skouterisb (2009); Crittenden y Woodside (2007); Dumitru (2012); Glevey (2006); Irany (2006); Moseleya et al. (2005); Murphya et al. (2013); Olaizola Rengifo (2007); Osman, Abdul Hamid y Hassan (2009); Pegram (2006); Saiz (2002); Sedaghat y Rahmani (2011); Serrano y Tormo (2000); Tebar Belmonte (2005); y el de Tesouro Cid (2006).

Finalmente, solo se encontraron dos artículos en grado de recomendación A (específicamente en nivel de evidencia I.a) y son los trabajos de: Abrami et al. (2008); y de Niu, Behar-Horenstein y Garvan (2013) (ver Tabla 6).

Tabla 6. Nivel de evidencia y grados de recomendación

\begin{tabular}{cccc}
\hline Variable de Análisis & Descripción de la variable & Frecuencia & Porcentaje \\
\hline \multirow{3}{*}{ Nivel de evidencia } & I.a & 2 & $3,3 \%$ \\
& I.b & 0 & $0,0 \%$ \\
& II.a & 4 & $6,7 \%$ \\
& II.b & 23 & $38,3 \%$ \\
& III & 10 & $16,7 \%$ \\
Grado de recomendación & IV & 21 & $35,0 \%$ \\
& Total & 60 & $100,0 \%$ \\
\hline & A & 2 & $3,3 \%$ \\
& B & 37 & $61,7 \%$ \\
& C & 21 & $35,0 \%$ \\
& Total & 60 & $100,0 \%$ \\
\hline
\end{tabular}




\section{DISCUSIÓN}

Cuando se analizan los resultados, lo primero que puede observarse es que la mayor parte de la producción académica sobre habilidades de pensamiento en el aula se publica en inglés. Esto puede indicar dos cosas que deben llevarnos al análisis: en primer lugar, que en los países en que hablamos español no hay tanto énfasis en las habilidades de pensamiento, o que sí lo hay, pero no estamos publicando en fuentes académicas nuestro desarrollo. Independientemente al motivo que lleve a esta situación, resulta preocupante que no estemos participando con suficiente empeño en una discusión central para el proceso educativo.

Lo segundo que llama la atención es que hay una tendencia en investigar el tema en términos empírico-analíticos y documentales. Esto indica que los investigadores deberían estar partiendo de constructos establecidos y adecuadamente delimitados para poder construir adecuadas deducciones. Sin embargo, habría que revisar con detenimiento en otro trabajo, si efectivamente los constructos teóricos que están utilizando los investigadores tienen la consistencia esperada y si hay puntos de acuerdo suficientes para conceptualizar el pensamiento.

Ahora, dentro de los estudios que no eran documentales, es importante diferenciar aquellos que se dedicaban a describir las habilidades de pensamiento de aquellos que proponían algún programa de estimulación de habilidades de pensamiento. Debido a los límites con la extensión del presente texto, los siguientes análisis se harán con los últimos.

Aunque desde hace mucho tiempo ya hay programas establecidos para la promoción del pensamiento (es el caso del Enriquecimiento Instrumental o el CASE), se observa que los autores tienden a poner en acción sus propios programas. Esta circunstancia ofrece una ventaja y una desventaja. La ventaja es que se está produciendo una variedad de programas de intervención que en general tiene buenos resultados, pero la desventaja es que no se están consolidando programas fuertes de trabajo que permitan orientar con claridad las acciones en el aula. Para ello, es recomendable hacer estudios sistemáticos que repliquen resultados de programas realizados por otros investigadores, de modo que se acumule evidencia sobre la funcionalidad de un programa en vez de información dispersa sobre distintos de ellos.

Además de la diversidad de programas, se observa que el diseño cuasi experimental ABA con grupo control es el que tienden a usar los investigadores para dar cuenta de su efectividad. Esto resulta adecuado, sin embargo, hay una limitación metodológica y dos procedimentales a tener en cuenta. Respecto a la metodológica, se observa que sumado a la diversidad de programas, hay también diversidad en los instrumentos para la evaluación de su eficacia (pruebas de inteligencia, de pensamiento y psicométricas); esto supone la dificultad de no poder comparar la eficacia de un programa con otro, haciendo aún más difícil la acumulación de evidencia.

Respecto a asuntos procedimentales de los programas, se hace evidente la tendencia a implementarlos por fuera del currículo, en periodos inferiores a seis meses y en gran medida en estudiantes de secundaria. Implementarlos por fuera del currículo puede hacer que no se aprovechen con suficiencia los contenidos curriculares que suponemos las habilidades de pensamiento facilitan y permiten transformar, además de correr el riesgo de convertirse en actividades extra para los estudiantes y los docentes, en vez de parte integral del proceso educativo. Que los programas duren menos de seis meses ofrece la dificultad de no tener un seguimiento a mediano o a largo plazo de los cambios que permite el programa y el posible 
impacto duradero en los procesos de aprendizaje. Se requieren estudios más largos que permitan una evaluación más profunda de los procedimientos. Además de lo anterior, que los programas se ejecuten sobre todo con población en secundaria, hace que no podamos conocer al detalle los impactos derivados una intervención temprana de las habilidades de pensamiento, que se ajuste una teorización que incluya periodos críticos de desarrollo.

Finalmente, se observa que en general los estudios clasificados con la Scottish tienen un grado de recomendación B o de evidencia moderada, probablemente las limitaciones metodológicas y procedimentales señaladas anteriormente tengan que ver en que no se logren niveles de evidencia mayores, pero sobre todo nos muestra que aún nos falta mucho trabajo si queremos consolidar las habilidades de pensamiento como un eje importante que optimice el proceso de aprendizaje.

\section{REFERENCIAS BIBLIOGRÁFICAS}

Abdullah, M., \& Osman, K. (2010). Scientific Inventive Thinking Skills among Primary Students in Brunei. Procedia - Social and Behavioral Sciences, 7, 294-301.

Abrami, P., Bernard, R., Borokhovski, E., Wade, A., Surkes, M., Tamin, R., \& Zhang, D. (2008). Instructional Interventions Affecting Critical Thinking Skills and Dispositions: A Stage 1 MetaAnalysis. Review of Educational Research, 78(4), 1102-1134.

Adey, P., Robertson, A., \& Venville, G. (2002). Effects of a cognitive acceleration programme on Year 1 pupils. British Journal of Educational Psychology, 72, 1-25.

Aizikovitsh, E., \& Amita, M. (2010). Evaluating an infusion approach to the teaching of critical thinking skills through mathematics. Procedia - Social and Behavioral Sciences, 2, 3818-3822.

Altintas, E., \& Sukru Ozdemir, A. (2012). The effect of teaching with the mathematics activity based on purdue model on critical thinking skills and mathematics problem solving attitudes of gifted and non-gifted students. Procedia - Social and Behavioral Sciences, 46, 853 - 857.

Amestoy de Sanchez, M. (2002). La investigación sobre el desarrollo y la enseñanza de las habilidades de pensamiento. REDIE. Revista Electrónica de Investigación Educativa, 4(1), 126-159.

Athman Ernst, J., \& Monroe, M. (2004). The effects of environment-based education on students' critical thinking skills and disposition toward critical thinking. Environmental Education Research, 10(4), 507-522.

Barak, M., Ben-Chaim, D., \& Zoller, U. (2007). Purposely teaching for the promotion of higher-order thinking skills: A case of critical thinking. Research in Science Education, 37, 353-369.

Bayona Vargas, J. (2003). La escuela: dejar pensar antes que enseñar a pensar. Educación y Educadores, 6, 237-240.

Beltran, M., \& Torres, N. (2009). Caracterización de habilidades de pensamiento crítico en estudiantes de educación media a través del test HCTAES. Zona Próxima, 11, 66-85.

Beyer, B. (2008). How to teach thinking skills in social studies and history. The Social Studies, 99(5), 196-201.

Black, B. (2012). An overview of a programme of research to support the assessment of Critical Thinking. Thinking Skills and Creativity, 7, 122-133.

Blattner, N., \& Frazier, C. (2002). Developing a performance-based assessment of students' critical thinking skills. Assessing Writing, 8, 47-64.

Borjas, M., \& De la Peña Leyva, F. (2009). Desarrollo de habilidades de pensamiento creativo en el área de Ciencias Naturales y Educación Ambiental. Zona próxima, 10, 12-35.

Bronwen, L., \& Howe, N. (2003). Solitary play and convergent and divergent thinking skills in preschool children. Early Childhood Research Quarterly, 18(1), 22-41.

Burke, L., \& Williams, J. (2008). Developing Young Thinkers: An intervention aimed to enhance 
children's thinking skills. Thinking Skills and Creativity, 3, 104-124.

Burke, L., \& Williams, J. (2012a). The impact of a thinking skills intervention on children's concepts of intelligence. Thinking Skills and Creativity, 7, 145-152

Burke, L., \& Williams, J. (2012b). Two thinking skills assessment approaches: "Assessment of Pupils' Thinking Skills" and "Individual Thinking Skills Assessments". Thinking Skills and Creativity, 7, 62-68.

Cerillo Martin, M. (2002). Enseñar a pensar: Desarrollo del pensamiento lógico. Aula Abierta, 80, 63-86.

Cherian, J., Rao, K., \& Srivastava, K. (2013). Developing critical thinking skills among secondary school students: Need of the hour. Golden Research Thoughts, 3(2), 1-3.

Colcotta, D., Russell, B., \& Skouterisb, H. (2009). Thinking about thinking: innovative pedagogy designed to foster thinking skills in junior primary classrooms. Teacher Development, 13(1), 17-27.

Crittenden, V., \& Woodside, A. (2007). Building Skills in Thinking: Toward a Pedagogy in Metathinking. Journal of Education for Business, 83(1), 37-43.

Cuicas, M., Debel Chourio, E., Cassadei Carnei, L., \& Alvarez Vargas, Z. (2007). El software matemático como herramienta para el desarrollo de habilidades del pensamiento y mejoramiento del aprendizaje de las matemáticas. Revista Electrónica Actualidades Investigativas en Educación, 7(2), 1-36.

Delicea, A., Ertekinb, E., Yazicib, E., \& Aydina, E. (2009). Preservice primary teachers' three dimensional thinking skills. Procedia - Social and Behavioral Sciences, 1, 2666-2672.

Demir, M. K. (2008). 2005 Social studies curriculum's effects on students' critical thinking skills. Egitim Arastirmalari-Eurasian Journal of Educational Research, 33, 113-126.

Deniz Yasgan, A., \& Kinkal, R. (2009). Investigating the relationship between formal operational thinking skills and science achievement of 7th and 8th Grade Primary School Students. The International Journal of Learning, 16(4), 417-434.

Dewey, J., \& Bento, J. (2009). Activating children's thinking skills (ACTS): The effects of an infusion approach to teaching thinking in primary schools. British Journal of Educational Psychology, $79,329-351$.

Dumitru, D. (2012). Critical thinking and integrated programs: The problem of transferability. Procedia - Social and Behavioral Sciences, 33, 143-147.

Garcia-Moriyon, F., Colom, R., Lora, S., Rivas, M., \& Traver, V. (2000). Valoración de filosofía para niños: Un programa de enseñar a pensar. Psicothema, 12(2), 207-211.

Glevey, K. (2006). Promoting thinking skills in education. London Review of Education, 4(3), 291302.

Irany, T. (2006). Teaching the critical thinking skill of explanation. The Agricultural Education Magazine, 78(6), 21-22.

Kaufman, R., \& Burden, R. (2004). Peer tutoring between young adults with severe and complex learning difficulties: The effects of mediation training with Feuerstein's Instrumental Enrichment programme. European Journal of Psychology of Education, 19(1), 107-117.

Kozulin, A., Lebeer, J., Madella-Noja, A., Gonzalez, F., Jeffrey, I., Rosenthal, N., \& Koslowsky, M. (2010). Cognitive modifiability of children with developmental disabilities: A multicentre study using Feuerstein's Instrumental Enrichment-Basic program. Research in Developmental Disabilities, 31(2), 551-559.

Larraz, R., \& Allueva, P. (2012). Efectos de un programa para desarrollar habilidades creativas. Electronic Journal of research in educational Psychology, 10(3), 1139-1158.

Mbano, N. (2003). The effects of a Cognitive Acceleration Intervention Programme on the performance of secondary school pupils in Malawi. International Journal of Science Education, 25(1), 71-81.

McLellan, R. (2006). The impact of motivational "world-view" on engagement in a Cognitive 
Acceleration programme. International Journal of Science Education, 28(7), 781-819.

Moseleya, D., Elliott, J., Gregsonc, M., \& Higgins, S. (2005). Thinking skills frameworks for use in education and training. British Educational Research Journal, 31(3), 367-390.

Murphya, C., Bianchib, L., McCullaghc, J., \& Kerr, K. (2013). Scaling up higher order thinking skills and personalcapabilities in primary science: Theory-into-policy-into-practice. Thinking Skills and Creativity, 10, 173-188.

Niu, L., Behar-Horenstein, L., \& Garvan, C. (2013). Do instructional interventions influence college students' critical thinking skills? A meta-analysis. Educational Research Review, 9, 114-128.

Olaizola Rengifo, C. (2007). Aprendiendo a pensar, dibujando. Theoria, 16(1), 23-30.

Olaoye, A. (2012). Cognitive Acceleration in Mathematics Education Lesson (CAMEL) in Nigeria. British Journal of Humanities and Social Sciences, 3(2), 77-86.

Osman, K., Abdul Hamid, S., \& Hassan, A. (2009). Standard setting: inserting domain of the 21st century thinking skills into the existing science curriculum in Malaysia. Procedia-Social and Behavioral Sciences, 1(1), 2573-2577.

Pegram, D. (2006). "What If?" Teaching research and creative-thinking skills through proposal writing. The English Journal, 95(4), 18-22.

Raiziene, S., \& Grigaite, B. (2005). Developing child's thinking skills by semantic mapping strategies. TRAMES, 9(2), 192-206.

Rendón Uribe, M. (2011). Incidencia de un programa de intervención pedagógica basado en habilidades de pensamiento crítico-reflexivo y aprendizaje cooperativo en la competencia socioemocional de estudiantes de la básica de la ciudad de Medellín. Revista Virtual Universidad Católica del Norte, 32. Recuperado desde: http://revistavirtual.ucn.edu.co/index.php/RevistaUCN/article/ view/28

Rezaei Kargar, F., Ajilchi, B., Kalantar Choreishi, M., \& Zohoori Zangene, Z. (2013a). The effect of teaching critical and creative thinking skills on the locus of control and psychological well-being in adolescents. Procedia - Social and Behavioral Sciences, 82, 51-56.

Rezaei Kargar, F., Ajilchi, B., Kalantar Choreyshi, M., \& Noohi, S. (2013b). Effect of creative and critical thinking skills teaching on identity styles and general health in adolescents. Procedia Social and Behavioral Sciences, 84, 464-469.

Saiz, C. (2002). Enseñar o aprender a pensar. Escritos de psicología, 6, 53-72.

Saleh, M. (2009). A mathematical program to develop the skills of thinking of children. Sistemics, Cybernetics and Informatics, 7(5), 84-90.

Sanz de Acedo Lizarraga, M., Sanz de Acedo Baquedanoa, M., Goicoa Mangadob, M., \& CardelleElawar, M. (2009). Enhancement of thinking skills: Effects of two intervention methods. Thinking Skills and Creativity, 4, 30-43.

Sarigoz, O. (2012). Assessment of the high school students critical thinking. Procedia-Social and Behavioral Sciences, 46, 5315-5319.

Sedaghat, M., \& Rahmani, S. (2011). A review of approaches to teaching thinking: appropriate approach for Iran education system. Procedia - Social and Behavioral Sciences, 30, 1037-1042.

Serrano, M., \& Tormo, R. (2000). Revisión de programas de desarrollo cognitivo. El programa de enriquecimiento instrumental. Relieve: Revista electrónica de investigación y evaluación educativa, 6(1), 1138-1194.

Sokol, A., Oget, D., Sonntag, M., \& Khomenkob, N. (2008). The development of inventive thinking skills in the upper secondary language classroom. Thinking Skills and Creativity, 3, 34-46.

Tebar Belmonte, L. (2005). Filosofía para niños de Mathew Lipman. Un análisis crítico y aportaciones metodológicas a partir del programa de enriquecimiento instrumental del profesor Reuven Feurestein. INDIVISA: Boletín de estudios de investigación, 6, 103-116.

Tesouro Cid, M. (2006). Enseñar a aprender a pensar en los centros educativos, incluso en las actividades de evaluación. Revista Electrónica Interuniversitaria de Formación del Profesorado, 9(1), 1-14. 
Tuncer, M., \& Ozeren, E. (2012). Prospective teachers' evaluations in terms of using reflective thinking skills to solve problems. Procedia - Social and Behavioral Sciences, 51, 666-671.

Ulas, A., Epcacan, C., \& Kocak, B. (2012). Assessment of the curriculum of Turkish language teaching in the 2 nd grade of primary education in terms of critical thinking skills. Procedia Social and Behavioral Sciences, 31, 369-375.

West, B. (2003). Student attitudes and the impact of GIS on thinking skills and motivation. Journal of Geography, 102, 267-274.

Ya-Ting, C., Ya-Chin Chuang, L., \& Shin-Shang, T. (2012). A blended learning environment for individualized English listening and speaking integrating critical thinking. Computers \& Education, 63, 285-305. 
